\title{
A pulsatile chest mass after Bentall procedure: a case report
}

\author{
Xiaofan Huang ${ }^{1}$, Dashuai Wang ${ }^{1}$, Yu Song ${ }^{1}$, and Long $\mathrm{Wu}^{1}$ \\ ${ }^{1}$ Affiliation not available
}

April 22, 2021

\begin{abstract}
Aortic dissection is an aggressive and life-threatening cardiac disease with highly challenging in surgical operation. Bentall procedure was potential complications. How to manage them would be important to improve patient outcomes. In this case, we present a 41-year-old male patient with iatrogenic aortic dissection. He had aortic valve replacement and repair of atrial septal defect in 2012. After 5 years, he suffered reoperation for aortic dissection. A year later the patient was readmitted for a voluminous pulsatile mass over the anterior thorax, confirming the presence of a huge pseudoaneurysm originating from the left coronary bottom performed during the Bentall procedure, requiring a third operation to repair the hemorrhagic site. The pseudoaneurysm was common complication after inclusion technique in Bentall procedure. How to effective hemostasis or tension-free anastomosis was important to improve patient outcomes.
\end{abstract}

\section{Introduction}

Aortic dissection is an aggressive and life-threatening cardiac disease with high morbidity and mortality. ${ }^{1,2}$ Risk factors for development of aortic dissection mainly include conditions associated with aortic media abnormalities and increased aortic wall stress such as hypertension and trauma. ${ }^{3}$ In addition, the incidence of iatrogenic aortic dissection may increase with the growing number of invasive vascular and cardiac procedures. ${ }^{4}$ Currently, the Bentall procedure in acute aortic dissection therapy was standard technique, but also remains highly challenging in emergency operation. ${ }^{2,5}$

\section{Case report}

Here, we present a 41-year-old male patient with iatrogenic aortic dissection. He had years of history of grade 3 hypertension. The initial cardiac surgery he underwent was aortic valve replacement and repair of atrial septal defect in Guizhou province in 2012. Follow-up computed tomography angiography in April 2014 indicated aneurysmal dilatation of the aortic root and aortic sinus. In 2015, he was diagnosed with chronic DeBakey type-I aortic dissection in Beijing, treated only with medical treatment.

The man was admitted to Wuhan Union Hospital through the emergency department on 31 December 2017, with a chief complaint of chest tightness and shortness of breath for 1 week. He suffered from persistent cough, dyspnea, orthopnoea, and bilateral lower limb edema after admission. Aortic computed tomography angiography indicated the formation of the DeBakey type-I aortic dissection and the ascending aortic aneurysm, with a maximum diameter of $12.1 * 12.6 \mathrm{~cm}$. And contrast medium was leaked from aortic to pulmonary artery. Emergency surgery was performed with right heart failure exacerbated rapidly. The formation of the aortic-pulmonary artery fistula and the ascending aortic dissection involving the right coronary ostium were observed during operation. A series of complicated procedures were performed including removal of the aortic valve prosthesis used in previous surgery, repair of the aortic-pulmonary artery fistula with bovine pericardial patch, composite root replacement using a NO.21 ATS composite valve-graft prosthesis, an end-to-side anastomosis between the left coronary ostium and the aortic graft, insertion of a stented elephant trunk into the descending aorta, reconstruction of aortic arch, wrapping around the graft 
using the native aneurysmal aortic wall and shunting the aneurysm sac to the right atrium. The entire operation lasted 11 hours. The patient recovered well after the operation and was discharged 14 days later.

The patient was readmitted to Wuhan Union Hospital through the emergency department on 28 October 2018, with a chief complaint of progressive chest pain and a pulsatile anterior chest mass for 1 week (Figure 1). Aortic computed tomography angiography revealed that the ascending aorta was surrounded by a large saccular mass of mixed densities, which eroded sternum. The saccular mass compressed trachea and pulmonary artery, pushing the heart down toward the left diaphragm (Figure 2). Emergency surgery was performed for the patient again. As the character of the mass remained unclear, femoral cannulation was performed, and the temperature was cooled down to $22^{\circ} \mathrm{C}$. The mass was found to be thrombus after the skin was cut open and a mass of thrombus were removed from the pericardial cavity. Active bleeding at the left coronary anastomosis was also observed. An $8 \mathrm{~mm}$ vascular prosthesis was anastomosed between the left coronary ostium and the ascending aorta prosthesis. Then the aortic clamp was released, and an off-pump right coronary artery bypass grafting was performed using the saphenous vein. The duration of the entire surgical procedure was 10.5 hours. The patient was reexamined with aortic computed tomography angiography showing no abnormalities and was discharged 11 days after the operation (Figure 3).

\section{Discussion}

We report a DeBakey type-I aortic dissection after aortic valve replacement occurring in a 41-year-old male patient. The second and third operations were extremely difficult as both were very complicated, and the patient's conditions were severe. Although the patient recovered well eventually, several issues deserve further discussion.

Aortic valve replacement remains the primary surgical treatment for aortic valve disease, however, it has been reported to be one of the independent predictors for the development of type-I aortic dissection. ${ }^{6}$ Aortic wall fragility, aortic wall thinning and aortic regurgitation were identified as independent risk factors of late dissection after aortic valve replacement in multivariate analysis, with associated probabilities of $22 \%, 7 \%$, and $14 \%$, respectively. ${ }^{7}$ Therefore, replacing both the aortic valve and ascending aorta may be a good prophylactic strategy for patients with more than one risk factor. ${ }^{7}$ In addition, it is worth mentioning that surgical techniques appear to be not associated with postoperative dissection as the prevalence of dissection after aortic valve replacement was constant over the years despite significant advances in surgical strategies. ${ }^{7}$

The composite valve-graft technique has been an important surgical strategy for aortic valve and root pathology since the original description by Hugh Bentall and De Bono in 1968 despite countless modifications. An inclusion technique was used as a generally accepted standard with the intrinsic aorta enclosing the graft for hemostatic purposes. An iatrogenic fistula from the periprosthetic space draining to the right atrium was introduced by Cabrol to decompress the false lumen. ${ }^{8}$ However, detachment or loosening of the anastomosis between the coronary ostium and the aortic graft can promote the formation of the pseudoaneurysm, a well-known complication of the Bentall procedure. ${ }^{9,} 10$

In this case, the continued expansion of the aneurysm sac penetrated the sternum, compressed the mediastinum and pulmonary artery, causing a series of symptoms, and thus a third surgery was required. The formation of the huge pseudoaneurysm was associated with the active bleeding of the left coronary anastomosis combined with the closure of the iatrogenic fistula. Effective hemostasis is the key to Bentall procedure; however, it is difficult to directly attach the coronary arteries to the aortic graft. ${ }^{8}$ Several modifications and refinements have been proposed to resolve these issues. Interposition of a segment vascular prosthesis from the aortic graft to the coronary ostia allows for a more tension-free anastomosis. ${ }^{11-13}$ Excision of a small cuff of surrounding aortic tissue as "coronary buttons" can considerably improve the visibility and facilitate the aortic-coronary anastomosis. Nowadays, the "button" modification of the original Bentall procedure has been a standard operative technique and is the one most widely used for aortic root replacement as the safety and long-term efficacy have been confirmed. ${ }^{14,15}$

In conclusion, an optimal treatment strategy should be based on a comprehensive assessment of the patient's condition. For aortic valve disease, whether to perform a single aortic valve replacement or with a simulta- 
neous aortic surgery for a prophylactic purpose should be carefully evaluated. Multiple surgical procedures developed to treat aortic root pathology are now at our disposal, in which the "button" modification of the Bentall procedure is currently most widely used. Nevertheless, no single surgical procedure is perfect. Individualized strategy should be highlighted because the specific anatomy of the patient demonstrated which operation was the most appropriate one.

\section{Conflict of interests}

The authors declare that there are no conflict of interests.

\section{Ethics approval and consent to participate}

This study was approved by the Ethical Committee of Tongji Medical College affiliated with Huazhong University of Science and Technology (06/09/2019 S054). The consent of patient has been obtained and the informed consent obtained was written.

\section{Author contribution}

$\mathrm{XH}$ collected and assembled of data and write the manuscript; DW write the manuscript and revised the manuscript; YS revised the manuscript and approve the manuscript; LW revised the manuscript. All authors have read and approved the manuscript.

\section{References}

1. Gudbjartsson T, Ahlsson A, Geirsson A, Gunn J, Hjortdal V, Jeppsson A et al. Acute type A aortic dissection - a review. Scand Cardiovasc J 2020;54:1-13.

2. Erbel R, Aboyans V, Boileau C, Bossone E, Bartolomeo RD, Eggebrecht H et al. 2014 ESC Guidelines on the diagnosis and treatment of aortic diseases: Document covering acute and chronic aortic diseases of the thoracic and abdominal aorta of the adult. The Task Force for the Diagnosis and Treatment of Aortic Diseases of the European Society of Cardiology (ESC). Eur Heart J 2014; 35 :2873-2926.

3. Bossone E, LaBounty TM, Eagle KA. Acute aortic syndromes: diagnosis and management, an update. Eur Heart J 2018; 39 :739-749.

4. Nunez-Gil IJ, Bautista D, Cerrato E, Salinas P, Varbella F, Omede P et al. Incidence, Management, and Immediate- and Long-Term Outcomes After Iatrogenic Aortic Dissection During Diagnostic or Interventional Coronary Procedures. Circulation 2015; 131 :2114-2119.

5. Sabe AA, Percy E, Kaneko T, Plichta RP, Hughes GC. When to Consider Deferral of Surgery in Acute Type A Aortic Dissection: A Review. Ann Thorac Surg 2020.

6. Pham N, Zaitoun H, Mohammed TL, DeLaPena-Almaguer E, Martinez F, Novaro GM et al. Complications of aortic valve surgery: manifestations at CT and MR imaging. Radiographics 2012; 32 :1873-1892.

7. von Kodolitsch Y, Loose R, Ostermeyer J, Aydin A, Koschyk DH, Haverich A et al. Proximal aortic dissection late after aortic valve surgery: 119 cases of a distinct clinical entity. The Thoracic and cardiovascular surgeon 2000; $48: 342$.

8. Cabrol C, Pavie A, Gandjbakhch I, Villemot JP, Guiraudon G, Laughlin L et al. Complete replacement of the ascending aorta with reimplantation of the coronary arteries: new surgical approach. $J$ Thorac Cardiovasc Surg 1981; $81: 309-315$.

9. Panos A, Amahzoune B, Robin J, Champsaur G, Ninet J. Influence of technique of coronary artery implantation on long-term results in composite aortic root replacement. Ann Thorac Surg 2001; 72 :14971501.

10. Niederhauser U, Rudiger H, Vogt P, Kunzli A, Zund G, Turina M. Composite graft replacement of the aortic root in acute dissection. Eur J Cardiothorac Surg 1998; 13 :144-150. 
11. Raanani E, David TE, Dellgren G, Armstrong S, Ivanov J, Feindel CM. Redo aortic root replacement: experience with 31 patients. Ann Thorac Surg2001; 71 :1460-1463.

12. Piehler JM, Pluth JR. Replacement of the ascending aorta and aortic valve with a composite graft in patients with nondisplaced coronary ostia. Ann Thorac Surg 1982; 33 :406-409.

13. Mills NL, Morgenstern DA, Gaudiani VA, Ordoyne F. "Legs" technique for management of widely separated coronary arteries during ascending aortic repair. Ann Thorac Surg 1996; 61 :869-874.

14. Westaby S, Katsumata T, Vaccari G. Aortic root replacement with coronary button re-implantation: low risk and predictable outcome. Eur J Cardiothorac Surg 2000; 17 :259-265.

15. Karangelis D, Tzertzemelis D, Demis AA, Economidou S, Panagiotou M. Eighteen years of clinical experience with a modification of the Bentall button technique for total root replacement. $J$ Thorac Dis $2018 ; 10: 6733-6741$. 

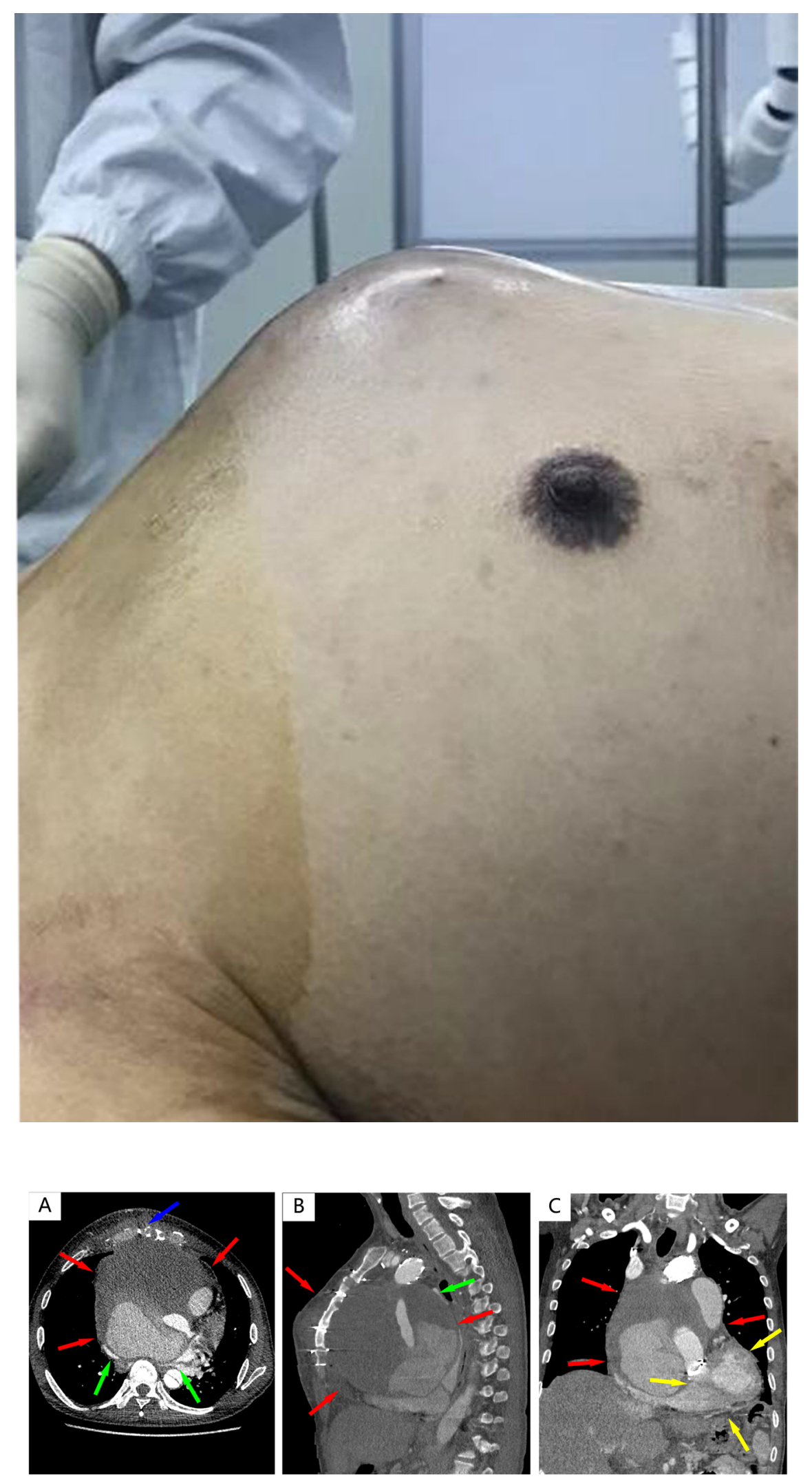

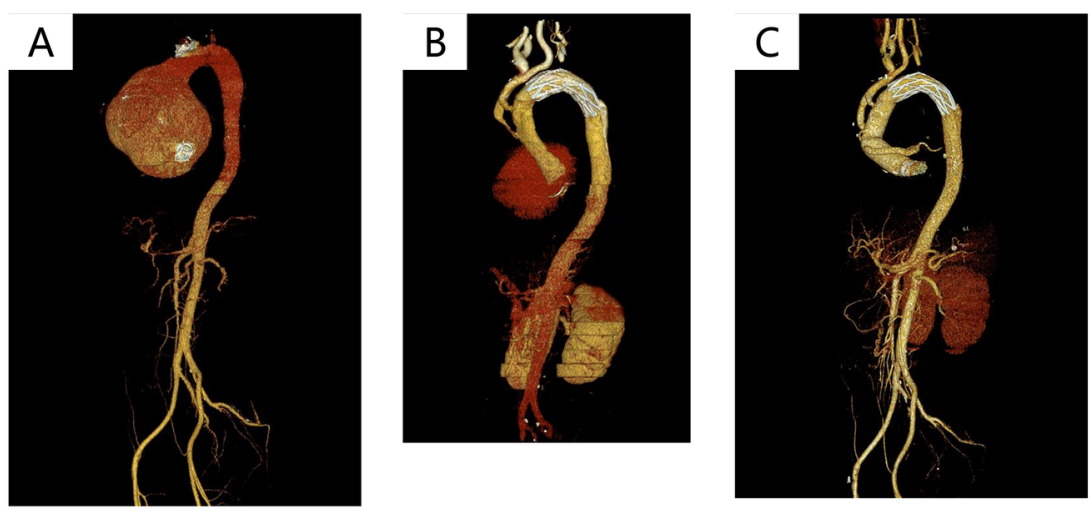